\title{
ESPECTROFOTOMETRIA DE LONGO CAMINHO ÓPTICO EM ESPECTROFOTÔMETRO DE DUPLO-FEIXE CONVENCIONAL: UMA ALTERNATIVA SIMPLES PARA INVESTIGAÇÕES DE AMOSTRAS COM DENSIDADE ÓPTICA MUITO BAIXA
}

\author{
André Luiz Galo* e Márcio Francisco Colombo \\ Departamento de Física, Instituto de Biociências, Letras e Ciências Exatas, Universidade Estadual Paulista "Júlio de Mesquita \\ Filho", Rua Cristóvão Colombo, 2265, 15054-000 São José do Rio Preto - SP, Brasil
}

Recebido em 27/2/08; aceito em 7/8/08; publicado na web em 26/1/09

\begin{abstract}
LONG OPTICAL PATH LENGTH SPECTROPHOTOMETRY IN CONVENTIONAL DOUBLE-BEAM SPECTROPHOTOMETERS: A SIMPLE ALTERNATIVE FOR INVESTIGATING SAMPLES OF VERY LOW OPTICAL DENSITY. We describe the design and tests of a set-up mounted in a conventional double beam spectrophotometer, which allows the determination of optical density of samples confined in a long liquid core waveguide (LCW) capillary. Very long optical path length can be achieved with capillary cell, allowing measurements of samples with very low optical densities. The device uses a custom optical concentrator optically coupled to LCW (TEFLON ${ }^{\circledR} \mathrm{AF}$ ). Optical density measurements, carried out using a LCW of $\sim 45 \mathrm{~cm}$, were in accordance with the Beer-Lambert Law. Thus, it was possible to analyze quantitatively samples at concentrations 45 fold lower than that regularly used in spectrophotometric measurements.
\end{abstract}

Keywords: liquid core waveguide (LCW); very long path length; light concentrator.

\section{INTRODUÇÃO}

A espectroscopia de absorção no UV-VIS tem ampla aplicação em laboratórios de análises e pesquisas físicas, químicas, bioquímicas, farmacológicas, etc. Inúmeras vantagens contribuem para sua popularidade; a principal, é o fato de ser uma técnica espectroscópica quantitativa. Aliado a isto, a técnica tem baixo custo operacional, é de fácil utilização e produz resultados de interpretação geralmente bastante simples. Em laboratórios analíticos, esta técnica é muito utilizada na quantificação direta de pequenas moléculas orgânicas e inorgânicas, de macromoléculas como proteínas e ácidos nucléicos ou na quantificação indireta de espécies inorgânicas, orgânicas e biológicas através da titulação de indicadores cromogênicos e/ou reagentes específicos. Sua utilização para pesquisa científica e tecnológica abrange uma ampla gama de áreas. Só para citar poucos exemplos, a espectroscopia de UV-VIS é utilizada desde a caracterização físico-química de reações químicas e bioquímicas, na descrição de mecanismos e cinéticas de reações biológicas complexas, na pesquisa de novos agentes farmacológicos até a investigação de propriedades óptico-eletrônicas de filmes finos de novos materiais.

A espectroscopia de absorção UV-VIS apresenta também limitações que são intrínsecas à sua qualidade quantitativa. A Figura 1 esquematiza a determinação quantitativa da absorção de luz por uma amostra, ou melhor, sua densidade óptica, na qual incide um feixe de luz de comprimento de onda $\lambda$ e intensidade $\mathrm{I}_{0}$; ao ser refratado, a intensidade do feixe decai exponencialmente com o caminho óptico $l$ percorrido no interior da amostra.

Sendo I, a intensidade do feixe emergente da amostra, a absorbância (A) ou densidade óptica da amostra é dada pela Equação 1, conhecida como Lei de Beer-Lambert (no caso de amostras constituídas por um único cromóforo)

$\mathrm{A}=-\log \left(\frac{I}{I_{o}}\right)=\varepsilon_{\lambda} \times C \times \ell$

*e-mail: galo@ibilce.unesp.br

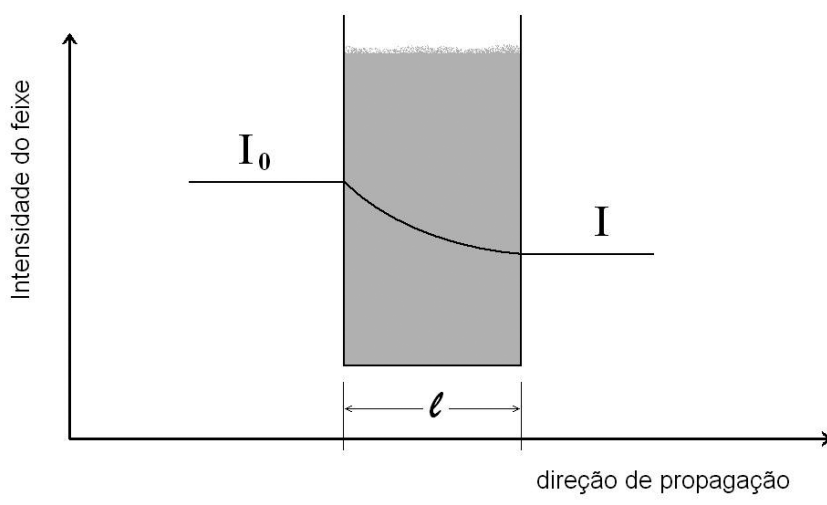

Figura 1. Diagrama representativo da absorção de luz por uma amostra em função da distância percorrida pela luz no interior da amostra (caminho óptico)

onde $\varepsilon_{\lambda}$ é o coeficiente de extinção molar da amostra para radiação de comprimento de onda $\lambda, l$ o caminho óptico e $C$ a concentração do cromóforo na amostra. No caso de amostras com n cromóforos distintos, a absorção total será a soma das contribuições individuais da absorbância de cada espécie $i$, isto é, $\mathrm{A}=\sum \varepsilon_{\lambda, i} \times C_{i} \times \ell$.

Essencialmente, estas expressões mostram que a medida da absorbância da amostra é proporcional a três quantidades: $\varepsilon_{\lambda}$ que é uma característica intrínseca às propriedades eletrônicas de cada cromóforo em cada comprimento de onda e aos parâmetros experimentais extrínsecos, a concentração do cromóforo e o caminho óptico percorrido pela luz na amostra. A maioria dos espectrofotômetros UV-VIS de duplo feixe apresentam boa linearidade na região de absorbância entre 0,01 e 3, enquanto que medidas com boa precisão são obtidas na faixa de absorbâncias entre 0,1 e 1 . Geralmente, estes limites são desimportantes quando se trabalha com amostras com alto coeficiente de extinção molar, e/ou disponíveis em quantidade. Entretanto, quando se depara com amostras com baixo coeficiente de extinção molar, ou disponíveis em concentrações muito baixas (quando, por exemplo, se 
deseja medir traços de concentração de um íon ou molécula dissolvida em um substrato que pode ser gasoso, líquido ou sólido), os limites de detecção característicos da espectroscopia UV-VIS podem requerer a utilização de amostras em cubetas com caminho óptico longo. Tipicamente, as cubetas para UV-VIS mais utilizadas têm caminhos ópticos de 5,10 e até $50 \mathrm{~mm}$, que requerem volumes de amostra entre $0,5,2$ e até $10 \mathrm{~mL}$, respectivamente. Portanto, a determinação espectrofotométrica da concentração de amostras com baixa absorbância pode ainda ser realizada utilizando-se porta amostras de caminho óptico longo. Para tanto, basta que haja disponibilidade em quantidade da amostra que se quer determinar. Em várias aplicações de interesse, tais como bioquímicas, biofísicas e farmacológicas, a regra é a baixa disponibilidade de material, devido tanto à dificuldade quanto ao alto custo envolvido na produção e purificação de amostras de enzimas, proteínas e ácidos nucléicos. Nestes casos não é raro considerar uma parca miligrama, como uma grande quantidade de material. Nestas condições, as limitações intrínsecas à espectroscopia UV-VIS tornam-se um fator limitante a seu uso.

Uma alternativa possível para a realização de medidas de absorção óptica de amostras com baixa concentração e/ou absortividade, utilizando-se pequenos volumes de amostra e caminho óptico longo, seria utilizar um porta-amostra capilar. Entretanto, esta alternativa apresenta algumas limitações. A primeira delas é que com o aumento do caminho óptico aumenta a probabilidade de espalhamento de luz pela amostra, condição na qual as medidas de absorbância deixam de ser quantitativas. Outra limitação está relacionada a dificuldades de realizar medidas de absorção em caminhos ópticos longos em espectrofotômetros comerciais. Nestes, a geometria dos feixes de luz tanto de prova quanto de referência está otimizada para aceitar cubetas de caminho óptico curto, entre 1 e $50 \mathrm{~mm}$. Mais especificamente, a geometria do feixe de luz é tal que ele está focado em uma área de aproximadamente $1 \times 10 \mathrm{~mm}$ localizada no centro do porta-amostra, onde as cubetas são posicionadas. Antes de atingir o foco o feixe é ligeiramente convergente, divergindo ligeiramente a partir deste ponto até atingir a fotomultiplicadora. Portanto, a utilização de um capilar como cela para medidas de caminho óptico longo, além de estar limitada por efeitos de espalhamento de luz, exige a refocalização do feixe de luz na abertura do capilar; além disto, a refocalização do feixe deve ser tal que a luz percorra um caminho paralelo ao capilar para evitar o desvio do feixe por refração do caminho da amostra e da fotomultiplicadora.

Este trabalho descreve detalhadamente a construção e testes de um dispositivo de longo caminho óptico (DLCO), que serve como um acessório a espectrofotômetros convencionais, constituído de dois concentradores de luz e um capilar de polímero inerte com índice de refração menor que o da água, que juntamente com uma solução aquosa, atua como um guia de ondas líquido. Devido ao índice de refração da solução de teste ser maior que o das paredes do capilar, o feixe de luz fica confinado ao interior da amostra por reflexão total da luz que incide na interface solução/capilar. Este dispositivo permite a realização de medidas de absorção óptica em caminho óptico longo (dezenas de $\mathrm{cm}$ até metros) utilizando pequenos volumes de amostra (tão baixos quanto alguns micro-litros). O dispositivo apresentado foi otimizado para ser utilizado em espectrofotômetros convencionais, facilmente disponíveis em diversos laboratórios de pesquisas.

\section{Espectrofotometria em guia de ondas de núcleo líquido}

Uma estratégia para obtenção de medidas de absorção de radiação por uma amostra ao longo de um caminho longo, utilizando um pequeno volume de amostra, é utilizar porta-amostra capilares. Neste caso, o volume amostral necessário é determinado pelo diâmetro do capilar e o volume interno de eventuais conexões. A realização de medidas com este tipo de cela requer, entretanto, que a intensidade da radiação que percorre a amostra não seja perdida por refração nas paredes do capilar. Recentemente, a DuPont ${ }^{\circledR}$ passou a produzir um fluoro-polímero amorfo, Teflon ${ }^{\circledR}$ AF 2400 , com índice de refração igual a 1,29, portanto, menor que o índice de refração da água. Nestas condições, toda a radiação proveniente de um meio aquoso que incide nas paredes do capilar recoberto com esta resina, em ângulos maiores que um ângulo limite (definido pela razão entre os índices de refração do Teflon ${ }^{\circledR} \mathrm{AF}$ e do meio aquoso), sofre reflexão interna total; esta condição permite a "construção" de uma fibra óptica líquida. Portanto, a utilização de um capilar de Teflon ${ }^{\circledR} \mathrm{AF}$, ou mesmo de um capilar de vidro ou qualquer outro material recoberto com este polímero minimiza perda de radiação do interior da fibra tanto devido ao espalhamento de luz, quanto devido a um não paralelismo do feixe de radiação de teste. Outra vantagem está na possibilidade de obter caminhos ópticos longos espiralando os guias de onda.

A utilização de tubos capilares de Teflon ${ }^{\circledR} \mathrm{AF}$ como guias de ondas de núcleo líquido (liquid capillary wave-guide, $\mathrm{LCW}$ ) para análises espectrais de absorbância e de fluorescência em meio líquido de baixíssimas concentrações tem sido reportada recentemente. ${ }^{1-4}$ Estes trabalhos pioneiros da aplicação de um LCW em espectrofotometria, como os de Byrne et al., ${ }^{1,2}$ utilizam um aparato óptico próprio, com sistemas de lentes e fibras ópticas convencionais, bem como fontes e detectores específicos e exclusivos para o sistema espectrofotométrico, o que torna o sistema relativamente oneroso e dedicado. Aqui propomos a montagem de um dispositivo que permite acoplar uma cela capilar LCW a um espectrofotômetro convencional, de maneira simples e de fácil execução. Neste sentido, nossa proposta é uma solução prática e de baixo custo, que permite uma ampla gama de análises. Sem prejuízo de outras aplicações, o nosso interesse em desenvolver este dispositivo está em investigar interações de alta e altíssima afinidade entre ligantes e macromoléculas biológicas cuja dificuldade está na determinação do ligante livre, onde as concentrações envolvidas na região de saturação são muito baixas. Outra grande vantagem deste sistema é o pequeno volume de amostra utilizado em uma medida.

\section{DESCRIÇÃO DO DISPOSITIVO}

O dispositivo óptico de longo caminho óptico (DLCO) é apresentado como um acessório composto por um concentrador óptico ("funil de luz") que coleta a luz emitida pela fonte do espectrofotômetro (Varian/Cary-3E) fazendo com que ela passe por um guia de ondas de núcleo líquido (tubo capilar de Teflon ${ }^{\circledR}$ AF 2400 - Biogeneral Inc., San Diego; d.i. $=0,56 \mathrm{~mm}$, d.e. $=0,80 \mathrm{~mm}$ ) antes de dirigir-se à fotomultiplicadora. O núcleo líquido do guia de ondas é a amostra de interesse. Dado o índice de refração do capilar $(1,29)$ e o da solução em seu interior $\left(1,33\right.$ se considerarmos água a $\left.20^{\circ} \mathrm{C}\right)$ o sistema capilar-amostra se comporta como uma fibra óptica convencional, onde reflexões internas totais prevalecem, confinando e conduzindo o feixe luminoso ao longo do capilar. Um diagrama esquemático do sistema óptico desenvolvido é mostrado na Figura 2. Outra grande vantagem do LCW de Teflon ${ }^{\circledR}$ AF é que apresenta uma baixa disper-

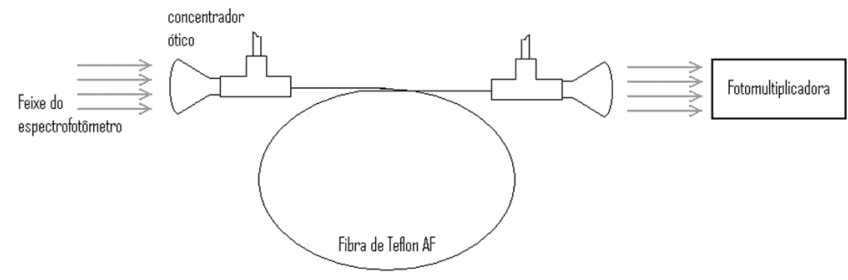

Figura 2. Representação esquemática do sistema óptico 
são luminosa para pequenas extensões, o que possibilita definirmos um caminho óptico de alguns centímetros até metros. Ainda que este dispositivo de longo caminho óptico tenha sido desenvolvido para ser acoplado em um espectrofotômetro Varian/Cary-3E, pode ser facilmente adaptado a qualquer espectrofotômetro similar. Uma fotografia deste dispositivo pode ser vista no Material Suplementar, Figura 1S.

\section{O concentrador óptico}

O concentrador óptico é peça fundamental do DLCO, pois possibilita a inserção do feixe no interior do LCW. Este concentrador foi construído em poli (metacrilato de metila) (PMMA) e sua geometria calculada de modo que todo feixe de luz incidente, dentro de um determinado ângulo de abertura, sofra reflexões internas totais até a sua outra extremidade, funcionando assim como um verdadeiro "funil de luz". O concentrador foi modelado segundo uma superfície esférica de raio $R$ na entrada, de forma que todo raio de luz incidente com um ângulo $<\phi_{1}$ em relação ao eixo óptico seja projetado no prolongamento do funil (saída do concentrador) obedecendo a condição de reflexão interna total $^{5}$ (Figura 3 ).

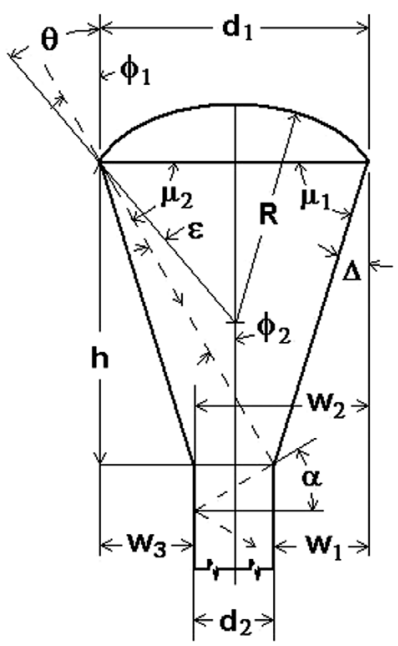

Figura 3. Geometria do concentrador e suas relações matemáticas com o "ângulo de entrada". As equações $W_{1} e W_{3}$ foram escritas relacionando as variáveis geométricas de forma que, para todo raio incidente dentro de um ângulo $\phi_{1}$, a condição de reflexão interna total seja obedecida. As relações e vínculos entre as variáveis que aparecem nestas equações são explicitadas na Tabela $1 S$

As relações entre as variáveis definidas na Figura 3 são mostradas na Tabela $1 \mathrm{~S}$ do material suplementar.

As relações geométricas mostradas na Figura 3 nos levam às equações:

$W_{1}=d_{1}-2\left\{d_{1}\left[\frac{\left(\sin \mu_{1}\right)\left(\sin \mu_{2}\right)}{\sin \left(\mu_{1}+\mu_{2}\right)}\right]\left[\tan \left(\frac{90-\alpha-\phi_{2}}{2}\right)\right]\right\}$

$W_{2}=h \tan \left\{\arcsin \left[\frac{\sin \left(\phi_{1}+\theta\right)}{n}\right]-\theta+2 \Delta\right\}$

$W_{3}=d_{1}-W_{2}$ e $W_{1}=h \tan \Delta$

O sistema é resolvido, uma vez que $\mathrm{R}, \theta$ e $\Delta$ possuem vínculos bem definidos (ver Tabela 1S), quando as condições $\mathrm{W}_{1}=\mathrm{W}_{3}$ e $\mathrm{d}_{2}=$ $\mathrm{d}_{1}-2 \mathrm{~W}_{1}$ são satisfeitas ${ }^{5}$ (Figura $2 \mathrm{~S}$, material suplementar). Entretanto, esta condição nos garante apenas que a luz proveniente da fonte do espectrofotômetro chegue à extremidade cilíndrica do funil (prolongamento). Outro fator essencial para um bom funcionamento do sistema é a minimização de perdas de potência luminosa no acoplamento funil/ fibra. O acoplamento, ou a focalização do feixe no interior da fibra, foi estudado e otimizado através de simulações computacionais. Várias geometrias distintas para a extremidade de saída do concentrador (acoplamento concentrador/LCW) foram estudadas a partir de simulações computacionais ray-tracing (Figura 4), com a finalidade de maximizar a potência luminosa transmitida ao LCW. Uma forma truncada rente ao cilindro na saída do concentrador aproveitaria apenas o fluxo integrado em uma área da ordem da área de uma secção transversal do LCW, o que tornaria o processo nada eficiente. Superfícies exclusivamente esféricas na saída do sistema não apresentaram bons resultados. A geometria apresentada, uma forma mista entre cônica e esférica (ver Figura 3S do material suplementar para maiores detalhes), além de proporcionar resultados satisfatórios é de fácil construção.

(A)

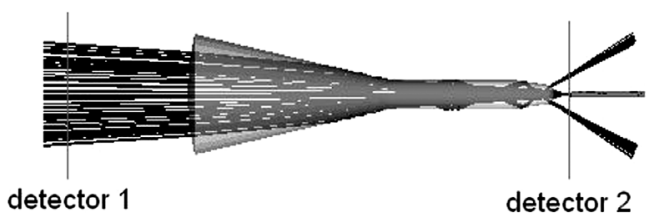

(B)
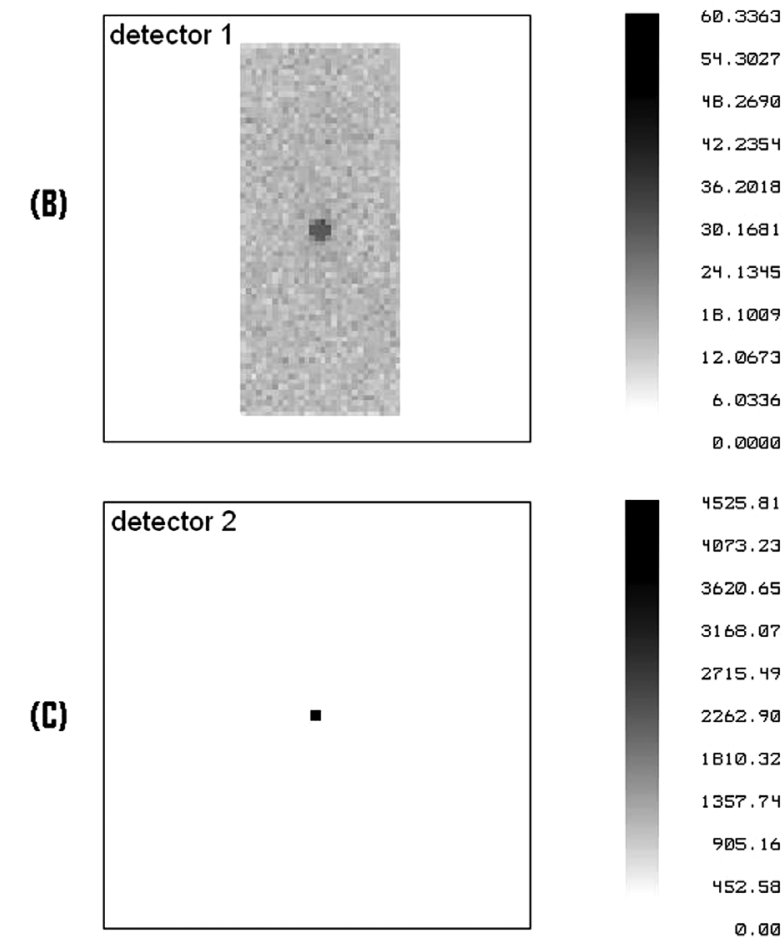

4525.81

4073.23

3620.65

$3168.0 ?$

2715.49

2262.90

1810.32

1357.74

905.16

452.58

0.00

Figura 4. Simulação ray-tracing do concentrador óptico. (A) Vista geral do comportamento do feixe ao longo do concentrador. Observamos que ofeixe é retangular e convergente ( $4^{\circ}$ em relação ao eixo óptico do sistema) de acordo com o perfil do feixe do espectrofotômetro Cary-3E. As reflexões/transmissões nas interfaces são determinadas pela diferença entre os índices de refração de cada meio, sendo que o feixe incidente contém $10^{6}$ raios luminosos. Um capilar com as dimensões e características do LCW utilizado foi posicionado na extremidade de "saída" do concentrador. (B) Distribuição transversal dos raios luminosos na "entrada" do funil. A escala de cinza (arbitrária) ao lado da figura indica a potência luminosa que atinge cada pixel do detector simulado (isto é, $n^{o}$ raios/área). (C) Distribuição transversal dos raios luminosos na "saída" do funil 
A Figura 4a mostra a simulação ray-tracing do formato final definido para nosso concentrador óptico. Nesta simulação, utilizamos um feixe retangular convergente (4 graus em relação ao eixo óptico do sistema, de acordo com o feixe do espectrofotômetro) representado pela trajetória de $10^{6}$ raios luminosos. A eficiência do funil é estimada por detectores transversais posicionados na entrada e na saída do funil, onde a potência luminosa é definida pela contagem do número de raios por unidade de área, isto é, número de raios incidentes em cada pixel do detector. As Figuras $4 \mathrm{~b}$ e $4 \mathrm{c}$ mostram a secção transversal do feixe que atinge um detector de $100 \times 100$ pixels na entrada e na saída do funil, onde uma escala arbitrária de potência luminosa é definida pela legenda ao lado da figura. Estas simulações mostram que a eficiência do concentrador é bastante razoável, ou seja, para uma potência fictícia de $1,000 \mathrm{~W}$ que atinge a entrada do funil (detector representado pela Figura $6 \mathrm{~b}$ ), 0,922 $\mathrm{W}$ atinge o segundo detector na saída (detector representado pela Figura 6c), sendo que 0,562 W atinge o interior da LCW (ponto central da Figura 6c). Na realidade, o perfil observado na saída do concentrador é um ponto focal cuja intensidade corresponde a $56 \%$ da intensidade total e um anel externo a este ponto, cuja maioria da intensidade é proveniente da região periférica da lente esférica na entrada do concentrador. Este fato é claramente visualizado projetando-se uma luz branca em um anteparo localizado próximo ao ponto focal do concentrador. Para compensar esta perda, bem como a atenuação intrínseca do LCW, instalamos um obturador no feixe de referência do espectrofotômetro.

\section{PARTE EXPERIMENTAL}

\section{Testes e calibração do DLCO}

Para testarmos o dispositivo e verificarmos a sua funcionabilidade, bem como o seu real potencial no estudo de interações biológicas de muito alta afinidade, realizamos uma série de medidas de absorbância com o corante murexida (diluído em água bi-destilada) e com uma droga de nosso interesse, Actinomicina-D (ActD, solução em tampão fosfato $10 \mathrm{mM}, 150 \mathrm{mM} \mathrm{NaCl}$ e $1 \mathrm{mM}$ EDTA), em baixíssimas concentrações. O corante murexida (ou purpurato de amônio) é uma substância bastante conhecida e comumente utilizada como indicador para análise de metais em compleximetria. $\mathrm{O}$ interessante é que uma solução de murexida possui um espectro de absorbância de forma gaussiana com absorção máxima em $520 \mathrm{~nm}\left(\varepsilon_{520}=1,4 \times 10^{4} \mathrm{~cm}^{-1} / \mathrm{M}\right)^{6}$ sendo bastante conveniente para o teste e calibração do DLCO. A droga ActD é um antibiótico natural que se intercala ao DNA natural apresentando um sítio de ligação de muito alta afinidade,,${ }^{7,8}$ motivação inicial para a construção deste dispositivo. A ActD livre apresenta um espectro de absorbância bem regular e conhecido, apresentando um máximo em $440 \mathrm{~nm}\left(\varepsilon_{440}=24800 \mathrm{~cm}^{-1} / \mathrm{M}\right){ }^{7}$ Em nossos testes, utilizamos dois caminhos ópticos distintos (comprimento do capilar de teflon), $32,27 \mathrm{~cm}$ e 46,5 cm e em todos os ensaios utilizamos um tempo de integração para a fotomultiplicadora de $0,333 \mathrm{~s}$.

O caminho óptico do sistema foi aferido a partir de medidas espectrofotométricas do corante murexida utilizando uma cubeta de quartzo de $10 \mathrm{~cm}$ de caminho óptico (Starna Cells, Inc.). Medidas de absorbância de várias soluções de murexida em diferentes concentrações foram realizadas utilizando-se o DLCO e a cubeta de $10 \mathrm{~cm}$. A partir da relação entre estas medidas é possível determinarmos com grande precisão o caminho óptico total do DLCO.

\section{RESULTADOS}

A atenuação intrínseca do DLCO $\left(I_{\mathrm{dB}}=10 \log \left(I / I_{0}\right)\right.$, Figura $4 \mathrm{~S}$ do material suplementar, foi determinada em relação à transmitância do sistema em $800 \mathrm{~nm}$ ( sinal de referência $I_{0}$ ). Tomando esta atenuação como linha de base, o sistema apresenta um espectro branco bem comportado na faixa de 400 a $800 \mathrm{~nm}$ (flutuações menores que 0,01 absorbância), porém, abaixo de $400 \mathrm{~nm}$ a intensidade do ruído aumenta demasiadamente, inviabilizando medidas confiáveis.

Espectros de absorbância de corantes (murexida, por exemplo) apresentaram uma relação sinal/ruído $(r)$ muito boa, sendo $r>100$ na faixa de 450 a $600 \mathrm{~nm}$ para sinais de 0,1 a 1 absorbância.

A Figura 5 mostra a correlação entre medidas de absorbância (em $520 \mathrm{~nm}$ ) realizadas com o DLCO e com uma cubeta de quartzo $(l=10 \mathrm{~cm})$ de amostras de murexida em diversas concentrações. $\mathrm{O}$ coeficiente angular deste gráfico fornece com grande precisão a proporcionalidade entre os dois caminhos ópticos (i,é., quanto a DLCO é maior que a cubeta de $10 \mathrm{~cm}$ ). Estes dados foram utilizados para a calibração do caminho óptico efetivo do DLCO.

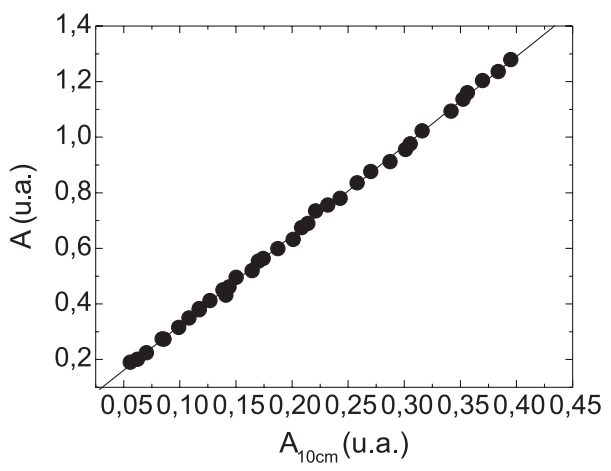

Figura 5. Correlação entre as medidas de absorbância da murexida (em 520 nm) com o DLCO e uma cubeta de quartzo (10 cm de caminho óptico). Cada ponto correlaciona medidas de uma mesma solução em ambos os dispositivos. Este gráfico nos dá a relação entre os dois caminhos ópticos (i.e, quanto o caminho óptico do DLCO é maior que o da cubeta). Do ajuste linear temos que o caminho óptico do DLCO é de 32,27 $\pm 0,06 \mathrm{~cm}$

A Figura 6 apresenta medidas de absorbância em $520 \mathrm{~nm}$ para o corante murexida em função da concentração. Tanto para a murexida quanto para a ActD, os resultados mostram uma excelente relação linear da absorbância com a concentração e, conseqüentemente, uma concordância muito boa com a lei de Beer-Lanbert. O coeficiente de extinção molar do corante murexida foi determinado como sendo $14134 \pm 164 \mathrm{~cm}^{-1} / \mathrm{M}$. Para a droga ActD livre, o coeficiente de extinção molar determinado foi de $24733 \pm 1220 \mathrm{~cm}^{-1} / \mathrm{M}$. Estes resultados

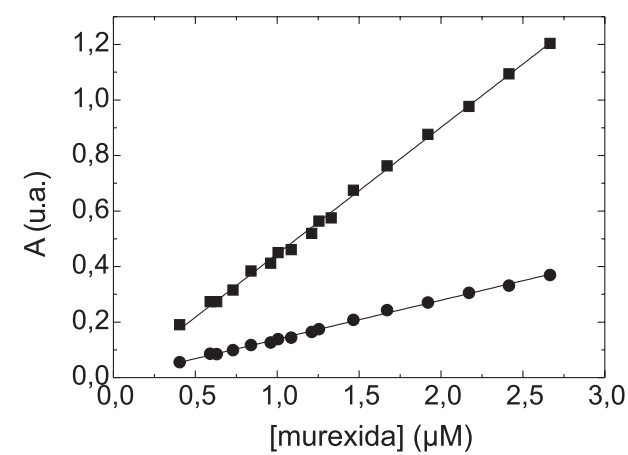

Figura 6. Gráfico de Bieer-Lanbert das medidas de absorbância em $520 \mathrm{~nm}$ do corante murexida no DLCO (•) e na cubeta de quartzo de $10 \mathrm{~cm}(\bullet)$. Os coeficientes de extinção molar, determinados a partir do DLCO e da cubeta de $10 \mathrm{~cm}$ são $14134 \pm 164 \mathrm{~cm}^{-1} / \mathrm{M}$ e $14043 \pm 177 \mathrm{~cm}^{-1} / \mathrm{M}$, respectivamente. Estes resultados estão de acordo com nosso valor de referência: $1,4 \times 10^{4}$ $\mathrm{cm}^{-1} / M^{6}$ 
apresentam uma grande concordância com os valores estabelecidos na literatura.

Espectros obtidos com o DLCO para a droga ActD utilizando um caminho óptico de $46,5 \mathrm{~cm}$ em comparação a espectros extraídos a partir de uma cubeta de quartzo de $1,0 \mathrm{~cm}$ de caminho óptico são mostrados no material suplementar (Figura 5S).

\section{CONCLUSÕES}

É sabido que o desempenho de todo o sistema óptico depende do comprimento de ondas que é utilizado. No caso de sistemas refratores, as propriedades ópticas dos materiais mudam com o comprimento de onda. Fenômenos associados às variações no índice de refração do material com o comprimento de ondas (dispersão, aberração cromática) bem como a variação do ângulo limite (máximo ângulo em que é possível a reflexão interna total) no concentrador e no interior do LCW interferem no desempenho do sistema óptico com um todo. Devido a estes fatores o sistema apresenta uma atenuação na intensidade que depende do comprimento de ondas e do comprimento $(l)$ do LCW aliada a uma perda de potência luminosa no acoplamento concentrador/LCW. Entretanto, a atenuação total da intensidade do sinal, ao menos dentro de uma faixa ótima, pode ser compensada pelo aumento do tempo de integração da fotomultiplicadora. Ao mesmo tempo, podemos melhorar a qualidade do sinal e minimizarmos os erros com a ofuscação do feixe de referência do espectrofotômetro através de um diafragma acoplado ao sistema compensando, assim, a absorbância intrínseca ao sistema como um todo.

Este sistema foi projetado para trabalhar na faixa espectral do visível (400 a $800 \mathrm{~nm}$ ) e, contudo, é penalizado devido às razões mencionadas anteriormente, em comprimento de onda abaixo de $400 \mathrm{~nm}$, onde o ruído se torna muito intenso e as perdas exageradas, inviabilizando a sua aplicação.

Entretanto, o dispositivo de longo caminho óptico apresenta grande linearidade na região do visível e registrou espectros muito bem definidos, com uma relação sinal ruído superando nossas expectativas.

Contudo, este sistema mostrou-se uma alternativa viável e de vasto potencial para o estudo de interações bioquímicas de muito alta afinidade, além de abrir várias outras possibilidades de aplicações. Outra grande vantagem deste dispositivo é o pequeno volume de amostra necessário para a realização de uma medida, geralmente quantidades inferiores a $1 \mathrm{~mL}$ são suficientes (volume máximo no interior do $\mathrm{LCW} \sim 100 \mu \mathrm{L}$ ), visto que para uma cubeta de $10 \mathrm{~cm}$ é necessário ao menos $20 \mathrm{~mL}$.

\section{MATERIAL SUPLEMENTAR}

A tabela e as figuras identificadas pelo complemento $\mathrm{S}(1 \mathrm{~S}$ a $5 \mathrm{~S})$ citadas ao longo do texto estão disponíveis gratuitamente em http:// quimicanova.sbq.org.br, na forma de arquivo PDF.

\section{AGRADECIMENTOS}

À Capes e ao CNPq pelo apoio financeiro. Agradecemos também, a Biogeneral Inc. (San Diego, USA) pelo fornecimento do capilar de Teflon ${ }^{\circledR} \mathrm{AF}$, peça fundamental para o desenvolvimento deste trabalho.

\section{REFERÊNCIAS}

1. Byrne, R. H.; Kaltenbacher, E.; Limnology and Oceanography 2001, 46, 740 .

2. Byrne, R. H.; Yao, W. S.; Kaltenbacher, E.; Waterbury, R. D.; Talanta 2000, 50, 1307

3. Keller, B. K.; DeGrandpre, M. D.; Palmer, C. P.; Sens. Actuators, B 2007, 125, 360 .

4. Wada, S.; Kawatsu, T.; Hashizume, Y.; Yabutai, T.; Motonaka, J.; Int. J. Modern Physics B 2006, 20, 25.

5. http://gltrs.grc.nasa.gov/reports/1997/CR-204145.pdf, acessada em Janeiro 2008 .

6. Ficher, M.; Knoche, W.; Robinson, B. H.; Wedderburn, J. H. M.; J. Chem. Soc., Faraday Trans. I 1979, 75, 119.

7. Ruggiero-Neto, J.; Colombo, M. F.; Biopolymers 2000, 53, 46.

8. Goodisman, J.; Rehfuss, R.; Ward, B.; Dabrowiak, J. C.; Biochemistry 1992, 31, 1046. 


\section{ESPECTROFOTOMETRIA DE LONGO CAMINHO ÓPTICO EM ESPECTROFOTÔMETRO DE DUPLO-FEIXE CONVENCIONAL: UMA ALTERNATIVA SIMPLES PARA INVESTIGAÇÕES DE AMOSTRAS COM DENSIDADE ÓPTICA MUITO BAIXA}

André Luiz Galo* e Márcio Francisco Colombo

Departamento de Física, Instituto de Biociências, Letras e Ciências Exatas, Universidade Estadual Paulista "Júlio de Mesquita Filho", Rua Cristóvão Colombo, 2265, 15054-000 São José do Rio Preto - SP, Brasil

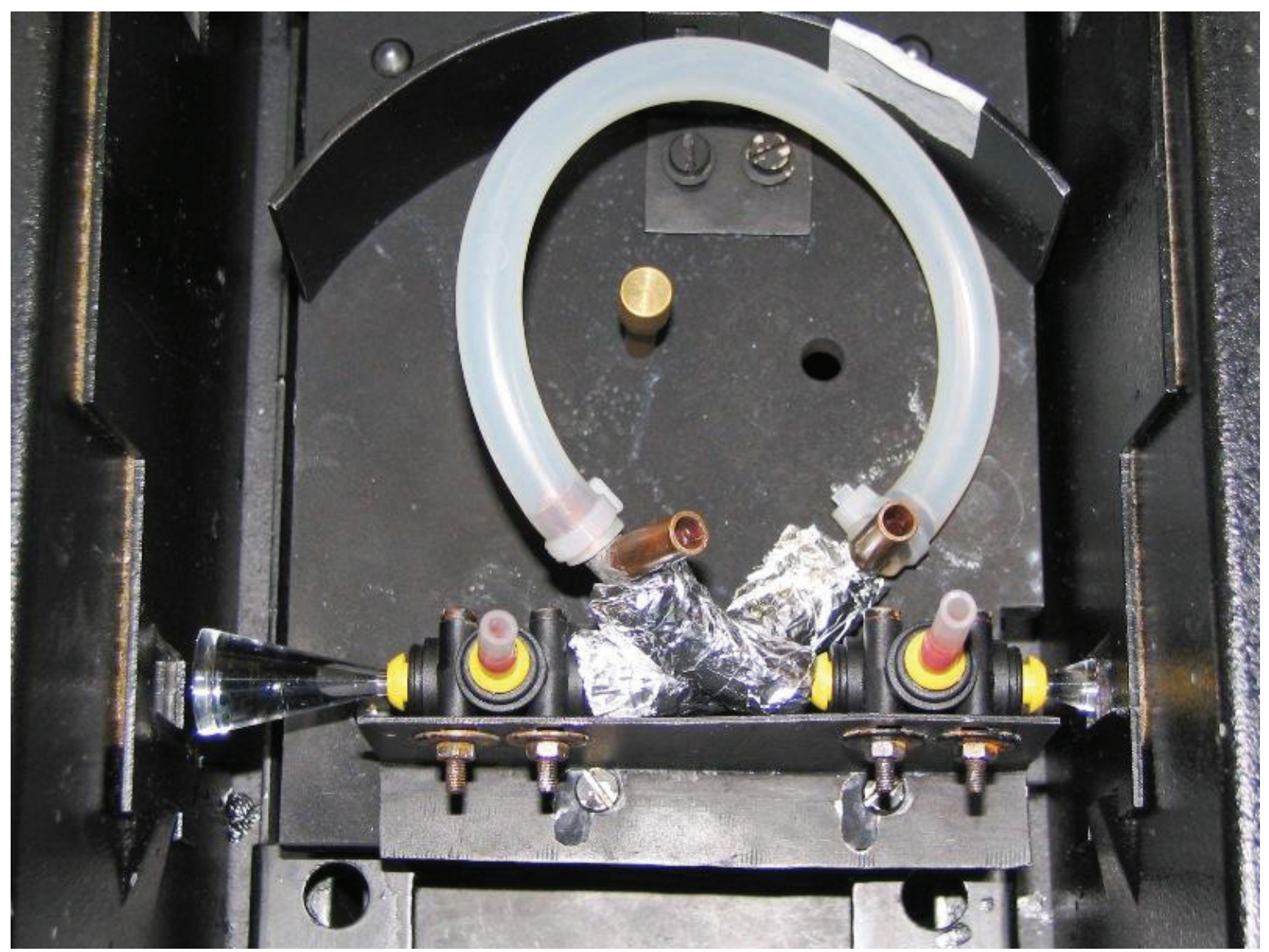

Figura 1S. Fotografia do dispositivo de longo caminho óptico acoplado a um espectrofotômetro Varian/Cary-3E. O LCW está encapsulado por uma mangueira de silicone que pode se conectada a um banho térmico 


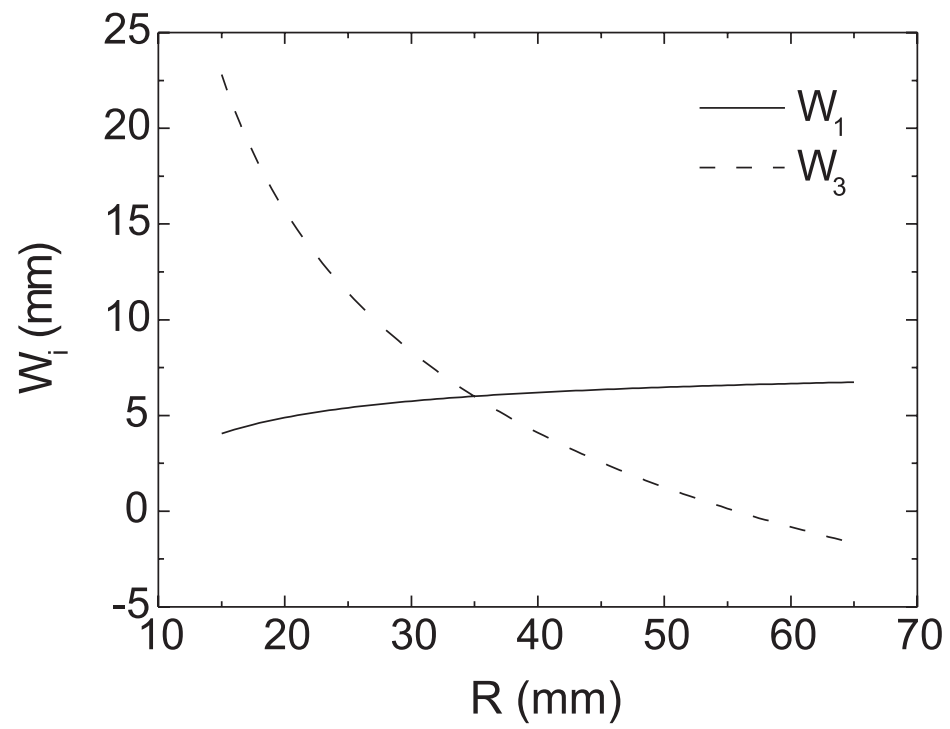

Figura $2 \mathrm{~S}$. $W_{1}$ e $W_{3}$ em função do raio da lente esférica do funil. $O$ sistema apresenta as soluções $R=34,96 \mathrm{~mm}, \theta=\Delta=13,23^{\circ}$ quando $W_{1}=W_{3}$
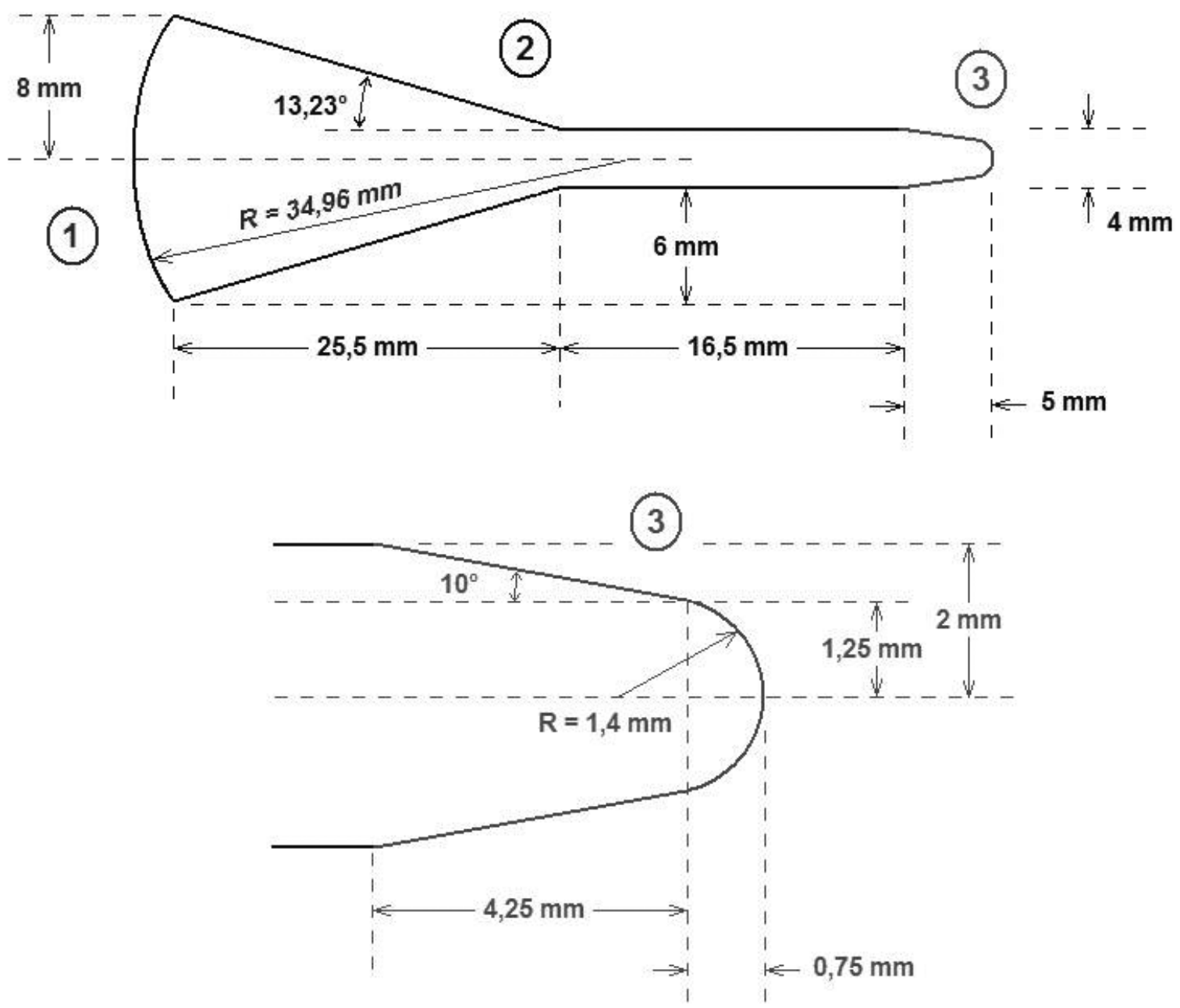

Figura 3S. (a) Dimensões determinadas para a construção do concentrador óptico. As distintas cores realçam a superfície de entrada (1), o cone principal (2) e a saída do concentrador (3). (b) Detalhe da saída do concentrador 


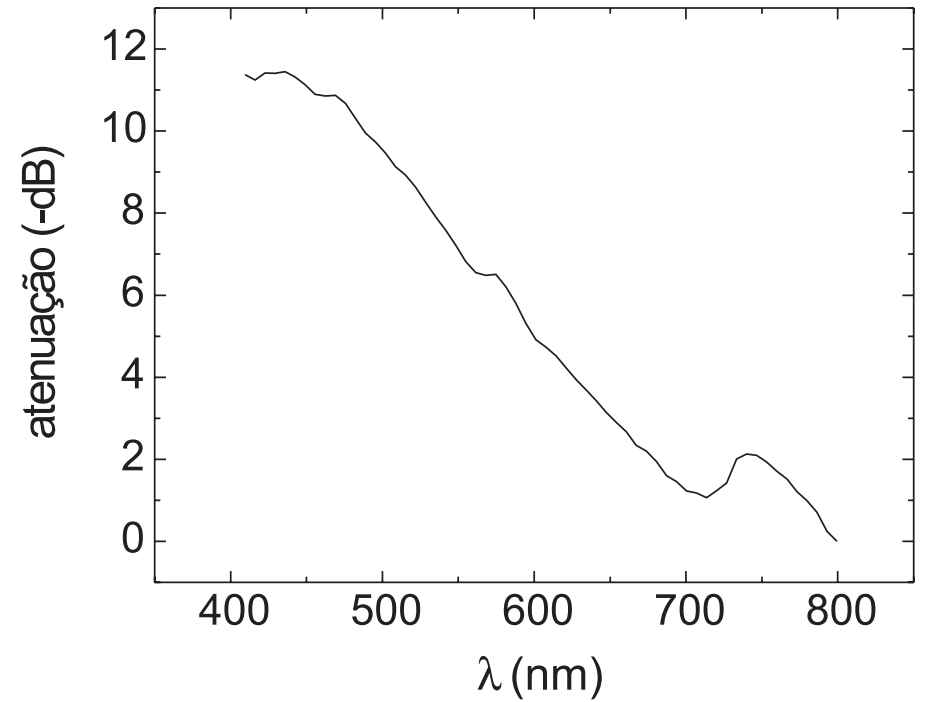

Figura 4S. Atenuação intrínseca do sistema óptico (concentrador $+L C W$ ) do dispositivo de longo caminho óptico. Este gráfico refere-se ao DLCO de 32,27 cm de caminho óptico analisando o sinal da água pura (bidestilada) e com tempo de integração de 0,333 s

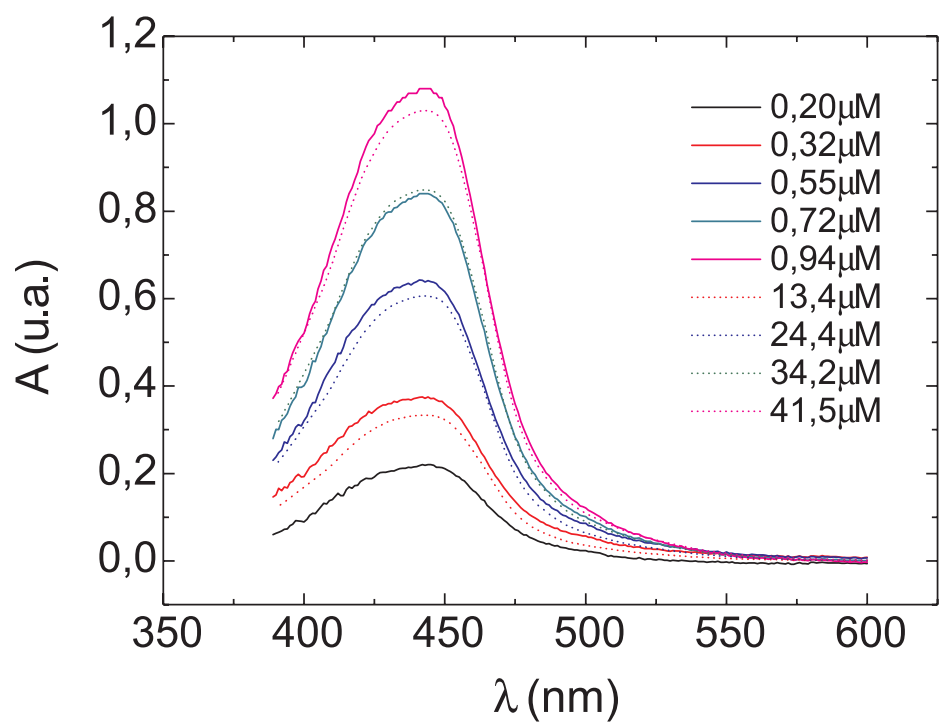

Figura 5S. Espectros de absorbância obtidos para a Act-D. Cada linha representa uma distinta concentração expressa em $\mu M$ na legenda. O caminho óptico utilizado foi de 46,5 cm e o tempo de integração da fotomultiplicadora foi de 0,333 s 
Tabela 1S. Relações entre as variáveis das equações $\mathrm{W}_{1}$ e $\mathrm{W}_{3}$ deduzidas a partir da geometria indicada pela Figura 3 (texto principal) tendo como vínculo principal a condição de reflexão interna total

$$
\begin{gathered}
\theta=\operatorname{arcsen}\left(\frac{\mathrm{d}_{1}}{2 \mathrm{R}}\right) \\
\varepsilon=\operatorname{arcsen}\left[\frac{\operatorname{sen}\left(\phi_{1}-\theta\right)}{\mathrm{n}}\right] \\
\phi_{2}=\theta+\varepsilon \\
\Delta=\frac{90-\alpha-\phi_{2}}{2} \\
\alpha=\operatorname{arcsen}\left[\frac{1}{\mathrm{n}}\right] \\
h=d_{1} \frac{\left(\operatorname{sen} \mu_{1}\right)\left(\operatorname{sen} \mu_{21}\right)}{\operatorname{sen}\left(\mu_{1}+\mu_{2}\right)} \\
\mu_{1}=h \tan \Delta \\
\Delta \text { and } \mu_{2}=90-\phi_{2}
\end{gathered}
$$

